\title{
Practice based education did not increase recognition of depression by primary care physicians nor improve the outcome of depression
}

\author{
Thompson C, Kinmonth AL, Stevens L, et al. Effects of a clinical-practice guideline and practice-based education on \\ detection and outcome of depression in primary care: Hampshire Depression Project randomised controlled trial. Lancet \\ 2000 Jan 15;355:185-91. \\ QUESTION: In primary care, is an education programme based on a clinical practice \\ guideline effective in improving the recognition and outcome of depression?
}

\section{Design}

Cluster randomised (allocation concealed*), unblinded,* controlled trial with 6 month follow up.

\section{Setting}

59 primary care practices in a health district in England, UK.

\section{Patients}

21409 patients attending the clinics of 152 physicians were screened. Exclusion criteria were being $<16$ years of age or too unwell to complete the questionnaire.

\section{Intervention}

29 practices (3 withdrew leaving 26 practices with 64 physicians) were allocated to the education group, and 30 (1 was excluded leaving 29 practices with 88 physicians) were allocated to the control group. Education using a clinical practice guideline (tricyclic antidepressants recommended as first line treatment and physicians advised to aim for a dose of $150 \mathrm{mg}$ ) was provided in 2 parts by a team. Part 1 consisted of 4 hours of seminars, in groups of up to 20 , at the beginning of the intervention year. If appropriate, teaching was supplemented by videotapes, small group discussions, and role playing. Part 2 consisted of the team being available for the next 9 months to provide additional information and help. Physicians in the control group started seminars 2 months after education had been completed in the intervention group.

Sources of funding: Medical Research Council; South and West Research and Development

Directorate;

Southampton

Community Health

Services Trust.

For correspondence: Professor C Thompson, Community Clinical Sciences Research

Division, Department of

Mental Health,

University of

Southampton, Royal

South Hants Hospital,

Brinton's Terrace,

Southampton

SO14 OPE, UK. Fax

$+44(0) 2380234243$.

Abstract and

commentary also

appear in

Evidence-Based Mental

Health.
Recognition of depression as defined by the Hospital Anxiety and Depression (HAD) scale, and clinical improvement of those identified as being depressed at 6 month follow up.

\section{Main results}

Analysis was by intention to treat. 4129 patients were classified as depressed by the HAD scale. The education and control groups did not differ in the sensitivity or specificity with which physicians recognised depression at any point during the study (after the seminars, after education, or at the end of the study). After education, the sensitivity and specificity with which physicians recognised depression was $39 \%$ and $92 \%$, respectively, in the education group compared with $36 \%$ and $93 \%$, respectively, in the control group $(\mathrm{p}=0.2$ for the difference between treatment groups). The improvement in depressed patients did not differ between the groups at 6 weeks or 6 months after the diagnostic assessment. The study had approximately an $80 \%$ power to detect a difference of $10 \%$ in clinical improvement between the treatment groups.

\section{Main outcome measures}

\section{Conclusion}

In primary care, a clinical practice guideline and practice based education did not increase physician recognition of depression nor improve the clinical outcome of those diagnosed.

*See glossary.

\section{COMMENTARY}

The evidence that education improves general practitioners' detection of depression is largely circumstantial, and the randomised controlled trial by Thompson et al attempts to counter the criticisms of previous investigations. By adopting a countywide approach to patients visiting the clinics of 152 physicians, a sufficient number $(>4000)$ were recruited to test the 2 main hypotheses. The authors sensibly included both the ability to detect depression successfully and the ability to relieve depression through effective treatment as outcome measures. Most previous investigations have concentrated only on the detection of depression. When the threshold for diagnosing depression is set below that which is clinically significant, failure to detect the symptoms may be unimportant.

The results of this study are convincingly negative. The difference of $3 \%$ in sensitivity between the groups is not large enough to be clinically important. I think this would be true even if different measures of depression were used.

It is therefore difficult to refute the conclusions of the authors that the offer of a clinical practice guideline along with additional education on treatment does not increase either the recognition of depression or success in improving its clinical outcome. We must recognise, however, that Thompson $e t$ al have chosen a topic that has been an issue for $>25$ years. The conclusions from this study do not necessarily imply that clinical practice guidelines and educational packages for other areas might not be more successful. Almost all doctors have known for a long time that people with depression present in many different ways in primary care and require adequate medication for at least 4 weeks before treatment can be effective. It is therefore possible that most general practitioners learnt this information in their training and the additional education offered in the experimental arm of this study added little to what they already knew. None the less, the findings are important because they should put a brake on optimism that educational initiatives alone can increase the recognition of depression in primary care and by extension reduce the national rate of suicide.

Peter Tyrer, MD Paterson Centre London, UK 